\title{
Wojciech Kunicki
}

Universität Wrocław, Wrocław

DOI: $10.19195 / 0435-5865.141 .4$

\section{Marian Szyrocki ${ }^{1}$ und Günter Grass ${ }^{2}$}

Eine wichtige Facette der Polen-Kontakte von Günter Grass stellt seine Beziehung zum Direktor der Breslauer Germanistik, Marian Szyrocki, dar. Im vorliegenden Aufsatz analysiere ich diese Kontakte aufgrund von unveröffentlichtem Briefwechsel zwischen Szyrocki und Grass sowie der IPN-Akten, die vor allem die Polen-Aufenthalte von Grass betreffen.

Der Verfasser der Blechtrommel erfreute sich zwar einer recht großen Beliebtheit, sein Hauptwerk durfte allerdings nicht offiziell erscheinen. Abgesehen von dem legendären Erstdruck in der Zeitschrift „Polityka“ brachte „Literatura na Świecie“ im Jahr 1976 einen umfangreichen Ausschnitt aus dem Roman, einige Aufsätze sowie ein Interview von Karol Sauerland mit Günter Grass. ${ }^{3}$ Allerdings war die Bekanntschaft und Freundschaft von Marian Szyrocki mit Günter Grass für das Profil der Germanistischen Studien (zumindest in Breslau) von großer Bedeutung. Diesen Kontakten möchten wir nun einige Worte anhand des Briefwechsels zwischen Günter Grass und Marian Szyrocki widmen. Die Kopien des Briefwechsels werden in der Akademie der Künste Berlin aufbewahrt und auch in der Bibliothek des Germanistischen Instituts der Universität Breslau, in der sie von Frau Krystyna Szyrocka deponiert wurden.

${ }^{1}$ Es sind nicht viele Beiträge, die sich mit den Kontakten von Marian Szyrocki mit deutschen Schriftstellern oder Professoren auseinandersetzen. Neben den unten zitierten Werken ist auf eine interessante, aber ziemlich apologetische Betrachtung von Karin Gafert hinzuweisen. Vgl. Karin Gafert: „Daß eine Nation die andere verstehen möge“ - Dieter Bänsch: ein schlesischer Brückenbauer. In: Zbliżenia interkulturowe. Polska. Niemcy. Europa. Interkulturelle Annäherungen. Polen. Deutschland. Europa. Polityka. Kultura. Społeczeństwo. Pismo Wyższej Szkoły Studiów Międzynarodowych w Łodzi. Łódź 07/2010, S. 67-78. Der Beitrag ist wertvoll wegen der Auswertung des ungedruckten Briefwechsels zwischen Marian Szyrocki und dem aus Niederschlesien stammenden Marburger Germanisten Dieter Bänsch.

2 Der Beitrag entstand im Rahmen des NCN/Projektes UMO-2011/03/B/HS2/04000

${ }^{3}$ Vgl. Literatura na Świecie 3/59 (1976). 
Den ersten Brief an Szyrocki schrieb Günter Grass am 6. Juni 1975. Das Schreiben hat den Adressaten offenbar nicht erreicht. Grass schickte darauf eine Abschrift als Anlage zum nächsten Brief vom 19. Januar 1976. Aus diesem Grunde ist die sich in der Bibliothek des Germanistischen Instituts befindliche Kopie das einzige Exemplar dieses Schreibens. Grass bekam die Adresse (Grunwaldzka 15 m. 72) von Albrecht Schöne. Sein Anliegen war mit der Arbeit am Roman Der Butt, an dem er seit zwei Jahren saß, verbunden. Die Handlung des Romans spiele im Mündungsgebiet der Weichsel. Auch die Stadt Danzig sei also auch präsent. Die Heldinnen sind elf Frauen, die ,jeweils in ihrer Zeit - vom Neolithikum bis in die Gegenwart - unauffällig gewirkt haben, denn die Geschichte, wie sie uns überliefert wird, wird geradezu ausschließlich von Männern gemacht und von Männern interpretiert.““4 Es folgt ein Katalog von zum Teil sehr detaillierten Fragen, die Grass beantwortet haben möchte, was vom hohen Ansehen Szyrockis als Kenner der barocken Literatur in der deutschen Kulturwelt zeugt. Grass hat eine ähnliche Intention wie der Wissenschaftler, möchte sie nur mit eigenen Mitteln verwirklichen: „Es reizt mich ungemein, auf meine fiktiv erzählende Art diese hier skizzierte Phase der deutschen Literatur freizuschaufeln und anschaulich zu machen." ${ }^{\text {5 }}$ Was aber dabei rauskommt ist die ursprüngliche Konzeption des Barockkapitels im Günter Grass: Der Butt: ${ }^{6}$

In Kürze, was mich hauptsächlich interessiert: Wo er in Danzig gewohnt hat, wie seine Lebensumstände gewesen sind, ob es die Möglichkeit einer (von mir inzwischen als Kapitel spekulierten) Begegnung zwischen Opitz und Gryphius Anfang des Jahres 1636 gegeben haben kann, ob er ein Liebesverhältnis in Danzig gehabt hat und ähnliche Fragen mehr, von deren Beantwortung ich mir einiges (wenn auch ungermanistische) Farbigkeit versprochen hatte. ${ }^{7}$

Grass kannte bereits die beiden Bücher von Szyrocki, insbesondere das Buch über Opitz, dem er „was die Schlußphase seines Lebens betrifft, zwar eine Fülle von Anregungen, aber doch nur wenige genaue Fakten entnehmen“" konnte. ${ }^{8}$

Nun möchte Grass von dem als besonders prominent geltenden Barockforscher Antworten auf seine sehr detaillierten Fragen bekommen.

Opitz wohnte bei dem Prediger Nigrinus. Wo befand sich dessen Haus? Stimmt es, dass Opitz in Danzig zu Beginn seines Aufenthaltes eine Liebesaffäre gehabt hat? Kann es sein, daß sich Andreas Gryphius und Opitz 1636 (als Gryphius abreiste, Opitz eintraf) in Danzig getroffen haben? Stimmt es, daß Hoffmannswaldau nach Opitz' Tod nach Danzig gekommen ist, um dessen literarischen Nachlaß zu übernehmen. Wo könnte ich in einige Originalbriefe, von Opitz an Oxenstierna gerichtet, einsehen? Gibt es von Opitz in Briefen oder anderen Zeugnissen Äußerungen über die Stadt Danzig und das Leben in ihr? Könnten Sie mir die Texte der beiden Opitzschen Schmähgedichte auf Magdeburg fotokopieren? Gibt es Hinweise auf Opitz`s Ge-

${ }^{4}$ Brief von Günter Grass an Marian Szyrocki vom 6. Juni 1975. In: Bibliothek des Instituts für Germanische Philologie der Universität Breslau.

${ }^{5}$ Ebd.

${ }^{6}$ Günter Grass: Der Butt.

${ }^{7}$ Brief von Günter Grass an Marian Szyrocki vom 19. Januar 1976. In: Bibliothek des Instituts für Germanische Philologie der Universität Breslau.

${ }^{8}$ Brief von Günter Grass an Marian Szyrocki vom 6. Juni 1975. In: Ebd. 
sundheitszustand während der Danziger Jahre oder kurz davor? Kann es sein, daß die drängenden Briefe von Opitz an Oxenstierna den schwedischen Kanzler dazu bewogen haben, militärisch wieder aktiv zu werden, die Armee Baners zu stärken? Und die kaiserlichen und die verbündeten sächsischen Truppen bei Wittstock (Oktober 1636) zu schlagen? Gibt es Anzeichen für einen Konflikt zwischen Opitz und seinem Vater, dessen Vitalität (viermalige Heirat) womöglich für den Sohn erdrückend gewesen ist? Sind überhaupt Verhältnisse von Opitz zu Frauen überliefert? Was hat es mit den unehelichen Kindern in Breslau auf sich? Gibt es kritische Äußerungen von Poeten der jüngeren Generation (Gryphius, Hoffmanswaldau und andere), die sich mit Opitz literarischem Werk und seiner politisch-diplomatischen Tätigkeit auseinandersetzten? Ist zu Opitz` Lebzeiten der Vorwurf des Opportunismus erhoben worden? Hat sich Opitz zu den in Danzig in deutscher Sprache geschriebenen Sonetten von Gryphius geäuBert. $^{9}$

Auf die beiden Briefe antwortete Szyrocki mit einem umfangreichen Brief am 25. Februar 1976, der m. E. viel dezidierter zur Erhellung mancher Sachverhalte, die Grass interessierten, beigetragen hatte, als die zweite, trotz der Notiz im Untertitel nur wenig veränderte Ausgabe des Buches über Martin Opitz, die 1974 bei C.H. Beck in München erschien. ${ }^{10}$ Erstens gibt es von Szyrocki (sehr hypothetische) Angaben, die von Grass nicht übernommen wurden. So lesen wir in dem Brief vom 25. Februar 1976 über den Wohnort von Nigrinus: „,nach einer polnischen Arbeit“ befand sich dessen Wohnung ,in der ulica Chlebownicza, was etwa „Brotgasse“ heißt“. Der Name der Gasse wird im Butt m.W. nicht erwähnt, dafür aber die sinnlichen Geräusche (,draussen wurden Fässer mit Eisenringen beschlagen“). ${ }^{11}$ Die Interpretation, „Opitz gehörte, wie es scheint, seit seiner Jugend dem „linken“ Flügel der Reformierten“ und in diesem Sinne auch seine doppelte Agenten-Tätigkeit als „Irenik (Friedensbemühungen)“12 verstand, wird von Grass bei der Schilderung des Treffens eher ironisch aufgefasst (in die modernen Kategorien übersetzt etwa als Verrat des Klerks gedeutet) ${ }^{13}$, in dem anschließenden Vortrag von Butt wird sie aber als eine ernstzunehmende Tatsache unterstrichen: „Ein weiteres Gutachten ortete den, bei allem scheinbar opportunistischen Wechsel, unverrückten politischen Standort des Dichters: Mitten im Dreißigjährigen Krieg sei er ein Ireniker gewesen. Eirene, das griechische Wort für Frieden, habe ihn bestimmt." ${ }^{14}$ Interessant ist auch, dass Szyrocki die Möglichkeit eines Treffens zwischen Opitz und Gryphius in Danzig bestreitet:

Andreas Gryphius und Opitz könnten sich während des Karnevals 1636 in Danzig getroffen haben. Opitz kam damals (höchstwahrscheinlich) in die Stadt, um dem Polnischen König Wla-

${ }^{9}$ Ebd.

${ }^{10}$ Marian Szyrocki: Martin Opitz. Zweite, überarbeitete Auflage. München 1974.

11 Günter Grass: Der Butt. S. 306.

12 Brief von Marian Szyrocki an Günter Grass vom 25. Februar 1976. In: Akademie der Künste. Briefwechsel Günter Grass-Marian Szyrocki. Berlin, Sign. 9047.

13 „All das tue Opitz gewiß in Sorge um das arme, schon wieder katholisch gepreßte Schlesien, aber auch um harte Taler, die ihm polnisch-schwedisch ausgezahlt worden für zwielichtigen Doppeldienst, für Spitzeltum und wieselige Zwischenträgerei“. In: Günter Grass: Der Butt. S. 308.

${ }^{14}$ Günter Grass: Der Butt. S. 322. 
dyslaw IV vorgestellt zu werden. Es scheint, daß Gryphius am 21. oder 22. Juli 1636 die Stadt verlassen hat, um nach Schlesien zurückzukehren. Opitz begab sich nach Danzig aber erst Ende August 1636. ${ }^{15}$

Grass hat sich dabei, um den ironischen Anschein der Gelehrsamkeit zu bewahren, eines fiktiven Zitats aus einem Brief an den Verleger Hühnerfeld bedient. ${ }^{16}$ Die stärkste Anregung konnte Grass in Sachen des Liebeslebens von Opitz von Szyrocki bekommen:

Die Liebesaffären, von Opitz wurden nie ganz aufgeklärt, es scheint aber, daß Opitz etwa 1629 fast gleichzeitig zweimal Vater wurde, wobei er sich für einige Zeit aus der Affäre zog, indem er eine Reise nach Frankreich unternahm. Über seine Flucht gibt es eine Reihe von Gedichten, die in einem bibliophilen Druck erschienen. Ich besitze diesen Druck, zwei von diesen Gedichten zitiere ich auf der Seite 140 meines Opitzbuches, zweite Auflage, München 1974. In Danzig verliebte sich Opitz in ein sehr junges Mädchen und wollte sie wohl heiraten, wie dies aus einem seiner Briefe zu ersehen ist. ${ }^{17}$

Davon ist im Butt nur der Vorwurf von Gryphius geblieben: „Und Breslauer Töchtern, man wisse von zwein, habe er durchreisend Kinder gemacht, aber die Alimente nicht zahlen wollen. " ${ }^{18}$ Das schwache Gegenargument von Opitz lautet: „habe sich mit Breslaus Töchtern mehr erschöpft als vergnügt.“19 Szyrocki weist auf die „Hässlichkeit“ von Opitz hin: „Opitz war häßlich, klein, abschreckend äuBerlich, doch anziehend im gesellschaftlichen Leben. (Schilderungen von Opitz in meinem Opitzbuch S. 69, Ausgabe Beck 1974). ${ }^{، 20}$ Bei Grass heißt es: „er lockerte den grämlichen Faltenwurf seiner Häßlichkeit zur Grimasse auf.“21

Grass webt in den Erzählduktus seines Romans fiktionale Episoden, die mit den authentischen Figuren von Martin Opitz, Anton Möller und Andreas Gryphius verbunden sind. Das Bindeglied zwischen dem Danziger Stadtmaler Möller und dem müden Dichter und Doppelagenten Martin Opitz ist die Köchin Agnes Kurbiella. Die Auseinandersetzung von Andreas Gryphius mit dem politisch fixierten und gelehrten, aber im Vergleich mit Gryphius poetisch weniger gewaltigen Martin Opitz bildet für Grass die Grundlage einer prinzipiellen Auseinandersetzung mit den halbherzig politischen und zugleich akademischen mittelmäßigen Dichtern und kann als solche auch eine zeitbezogene Bedeutung haben. Das bestätigt auch der Butt in seinem Schlussvortrag zu Opitz, in dem er viele (germanistische) „Gutachter“ herangezogen hat. Der Butt betont hier ironisch die „emanzipatorische“ Bedeutung seines

15 Brief von Marian Szyrocki an Günter Grass vom 25. Februar 1976. In: Akademie der Künste. Briefwechsel Günter Grass...

16 Günter Grass: Der Butt. S. 306.

${ }^{17}$ Brief von Marian Szyrocki an Günter Grass vom 12. Februar 1976.

18 Günter Grass: Der Butt, S. 309.

19 Ebd. S. 310. Bei Szyrocki handelt er mit dem Hinweis auf die zwei Gedichte um die in der zweiten Auflage erweiterte Fußnote 62 unter Berufung auf den Aufsatz von Janis L. Gellinek: Liebesgedichte und Lebensgeschichte bei M. Opitz, in: Deutsche Vierteljahresschrift für Literaturwissenschaft und Geistesgeschichte 42 (1968), S. 161-181.

${ }^{20}$ Brief von Marian Szyrocki an Günter Grass vom 12. Februar 1976. Diese Beschreibung ist auch eine der Ergänzungen in der zweiten Auflage des Opitz-Buches.

${ }^{21}$ Günter Grass: Der Butt, S. 312. 
Buches von der deutschen Poeterey, den Irenismus seiner nach Ausgleich zwischen den Religionen suchenden Bestrebungen, auch seinen Einfluss auf das Werk des von Grass geliebten und produktiv verarbeiteten Grimmelshausen. ${ }^{22}$ Man kann selbstverständlich Bezüge vor allem zwischen der Monographie von Szyrocki und den Darstellungen und den Wertungen von Grass ausfindig machen, ${ }^{23}$ allerdings sind das, wie Grass in einem seiner Briefe formulierte, eher „Anregungen“ und weniger konkrete oder gar wörtliche Bezüge. Es ist nämlich unübersehbar, dass Szyrocki merkwürdigerweise bei seinen Wertungen weniger den Kosmopolitismus von Opitz betonte, viel stärker aber seine „nationale“ Haltung und auch seine Bedeutung für die „nationale“ Poesie der Deutschen. Über die Breslauer Ausgabe der Opitzschen Gedichte schreibt Szyrocki zum Beispiel: „daß sie trotzdem bedeutend zur Bildung der gesamtnationalen deutschen Sprache und Dichtung beigetragen hat." ${ }^{24}$ Über das Buch von der deutschen Poeterey heißt es bei ihm: „Es wies den deutschen Dichtern den Weg zur Literatur in der Muttersprache und trug zur allmählichen Verdrängung der neulateinischen Poesie bei; es schuf den Ausgangspunkt für die deutsche Nationalliteratur.“25 Zu Anfang seines Buches spricht Szyrocki von Schlesien als einem Land, in dem sich „Anfänge einer Literatur in Nationalsprachen“ zeigen: er meint dabei das 1612 herausgegebene Werk des polnischen Dichters Walentin Rozdzienski Officina Ferraria (Über die Hütte) und „das 1617 geschriebene erste Werk Opitzens gegen die Verachtung der deutschen Sprache." ${ }^{26}$ Szyrocki betont auch die Rolle Schlesiens bei der Herausbildung der ,deutschen Nationalliteratur““ ${ }^{27}$ Diese Töne waren nichts Ungewöhnliches in der polnischen, durch den Marxismus durchsetzten (aber nicht marxistischen!) Sprache der Wissenschaft, die Szyrocki hier übernimmt und praktiziert, ungewöhnlich war der Zungenschlag bei den westdeutschen Intellektuellen, die einerseits auf Szyrockis Unvoreingenommenheit mit Bewunderung schauten (auch dort, wo er von der ,,schlesischen Heimat“ ${ }^{\text {“28 }}$ und Heimatliebe spricht), andererseits standen sie diesen und ähnlichen nationalgefärbten Formulierungen denkbar fern, wie zum Beispiel Günter Grass, der Szyrocki zwar „Anregungen“, aber keine genauen „Angaben“ verdankte. Man bemerke, dass der Grassche Opitz ein Inbegriff eines modernen Dichters ist und das Problematische seiner Haltung sich im Verhältnis zur Macht (und nicht etwa zu den kulturellen

22 Günter Grass: Der Butt. Roman, Darmstadt und Neuwied 1977, S. 322-323. Das konnte Grass einer Monographie von Christoph Stoll: Hans Jacob Christoffel von Grimmelshausen, 1676/1976. München 1976 entnehmen. Früher noch haben sich andere auch damit beschäftigt. Vgl.: Hans Geulen: Arcadische Simplizissiana. Zu einer Quelle Grimmelshausesns und ihrer strukturellen Bedeutung für seinen Roman, in: Euphorion 63 (1969), S. 426-37. Zur Problematik Grass und der barocke Dichtung siehe vor allem: Alexander Weber: Günter Grass `s Use of Baroque Literature. London 1995.

${ }^{23}$ Vgl. Alexander Weber: ebd., S. 9, 78, 84-88, 90, 91, 120, 122. Alle diese Belege sind überbewertet.

${ }^{24}$ Marian Szyrocki: Martin Opitz, S. 72.

25 Ebd., S. 68.

${ }^{26}$ Ebd., S. 9.

27 Ebd., S. 10. Dasselbe wird noch stärker auf S. 11 wiederholt.

28 So wörtlich in: Marian Szyrocki: Der junge Gryphius. Berlin 1959, S. 129. 
Leistungen auch für das Nationalbewusstsein der Deutschen, das es damals so wie im 19. und 20. Jahrhundert nicht gab) kristallisiert. ${ }^{29}$

Die Kontakte waren sporadisch, aber herzlich. Die beiden hatten sich offensichtlich bereits 1960 bei dem ersten Aufenthalt von Marian Szyrocki in der Bundesrepublik kennengelernt: „Ich lernte Sie bereits 1960 in Bonn und Aschaffenburg kennen, und nach sehr vielen Wodkas haben wir sogar geduzt. ${ }^{\text {‘30 }}$ Wie es scheint, war der Hauptberater von Günter Grass Albrecht Schöne, exzellenter Kenner der deutschen Barockdichtung. Am 10. Dezember 1976 sandte Szyrocki herzliche Grüße an Grass mit der Einladung nach Breslau zu kommen:

Lieber Herr Grass, bei uns schneit es seit Tagen und wir laufen in Pelze gehüllt, mit Füchsen auf den Köpfen durch die Straßen. In dieser kalten Atmosphäre gedenke ich Ihrer im Kollektiv von 666 Studentinnen unseres Instituts, die Ihnen alle frohe Festtage und ein glücksschwanegeres Jahr 1977 wünschen. In der Hoffnung, Sie bald in der Stadt zu sehen, in der der Brotherr von Agnes Korbiella (sic!) die ärgsten Sünden begangen hat, verbleibe ich mit besten Grüßen Ihr. ${ }^{31}$

Am 3. April 1979 bittet Szyrocki um ein Exemplar des Romans Das Treffen in Telgte, der ihm vom Büro Grass (Eva Hönisch) am 6. April 1979 geschickt wurde. Der Dichter selbst hielt sich in Alaska auf. ${ }^{32} \mathrm{Zu}$ einer Intensivierung der Kontakte zwischen beiden kam es erst Mitte der 80er Jahre. Nach einer Absprache mit dem Dichter in Göttingen während des IVG-Kongresses ${ }^{33}$ setzte ihn Szyrocki „auf unsere Gästeliste gesetzt“ und ging davon aus, dass er „etwa im Februar (1986) eine Einladung an unsere Universität erhalten" würde. ${ }^{34}$ In demselben Brief teilt er Günter Grass mit, dass sich in seinem Magisterseminar zehn Studenten mit „Ihrer Lyrik" beschäftigen werden. Für das Sommersemester bekam er eine Einladung nach München und dort würde er ein Hauptseminar zur Lyrik von Günter Grass halten. „Bei dieser Arbeit häufen sich Fragen und Zweifel. Ich hoffe, daß ich Sie

${ }^{29}$ Szyrocki konnte sich so etwas leisten bei gleichzeitigen Hinweisen auf den ,polnischen Charakter"Schlesiens, was er in seinem Gryphius-Buch (1959), und zwar in seinen Anfangspartien, noch stärker betonen wird.

${ }^{30}$ Brief von Marian Szyrocki an Günter Grass vom 12. Dezember 1975. In: Akademie der Künste. Briefwechsel Günter Grass-Marian Szyrocki. Berlin, Sign. 9047.

31 Brief von Marian Szyrocki an Günter Grass vom 18. Dezember 1977. In: Bibliothek des Instituts für Germanische Philologie der Universität Breslau. Szyrocki hätte bei einer genaueren Lektüre des Barock-Kapitels zumindest den falschen Namen des polnischen Königs korrigiert, der lautet nämlich an einer Stelle nicht Wladyslaw, sondern „Wladimir, den Polenkönig“ (Günter Grass: Der Butt, ebd., S. 352). Ebenfalls hätte er korrigieren können, dass Raffael Leszczynski kein „Fürst“ war, wie Grass im Butt auf S. 307 behauptet.

${ }^{32}$ Brief von Eva Hönisch an Marian Szyrocki. In: Bibliothek des Instituts für Germanische Philologie der Universität Breslau.

33 Günter Grass las dort einige Ausschnitte aus dem neuen Roman Die Rättin vor.

34 Man muss betonen, dass Grass 1985 kein Einreisevisum nach Polen bekam wegen seiner Kritik des Kriegszustandes. Seit dieser Zeit bemerkt er: ich bin alle drei Jahre in Polen, „wenn ich eingelassen werde". 
dann in Polen befragen kann. Inzwischen lese ich meinen Assistenten und Studenten die „Rätin“-Kapitel aus der »Zeit«.“35

Das Magisterseminar zu Günter Grass war nicht der Lyrik, sondern verschiedenen Aspekten des Werkes insgesamt gewidmet, Szyrocki hatte es 1986 initiiert, zur Zeit der Abfassung des Briefes war das erst eine Absicht. Es kam aber zustande und in zehn Magisterarbeiten wurden verschiedene Aspekte der Werke von Grass herausgearbeitet. ${ }^{36}$ Es ist durchaus möglich, dass Szyrocki gerade mit den in das Werk von Grass eingelesenen Studentinnen den Lyrik-Workshop plante, von dem er im nächsten Brief an Grass berichtete. Am 28. Februar 1986 ging eine offizielle Einladung an Grass, von dem Prorektor der Universität, Norbert Morciniec, unterzeichnet. Morciniec knüpfte auch an das Lyrik-Vorhaben Szyrockis an. Im Begleitbrief Szyrockis vom 4. März 1986 heißt es:

Das Kolloquium stelle ich mir etwa so vor: Nach einer kurzen Einführung zum Thema würde ich Sie bitten, jeweils ein Gedicht von Ihnen zu lesen, dem im Anschluß eine kurze, sehr sachliche Interpretation folgen würde. Nach einer Diskussion - ich wäre Ihnen für kritische Bemerkungen dankbar - würden wir zum nächsten Gedicht übergehen. Ich schlage vor, dass wir dieses Arbeitsgespräch während zwei Doppelstunden (90 Minuten) am Vormittag und Nachmittag bzw. an zwei verschiedenen Tagen abwickeln, um die polnischen Zuhörer nicht zu überfordern. ${ }^{37}$

Die Gedichte habe er ausgesucht; die Kurzinterpretationen könnte er Grass entweder zuschicken oder „bei der Ankunft überreichen“. Das Kolloquium sollte im kleinen Kreis abgewickelt werden (ca. 50 Personen), „doch Sie wissen, dass wir uns eventuell den Zwängen des Andrangs beugen müssen.“38 „Ich biete mich an, Sie durch die Stadt zu führen, Ihnen die Wirkungsstätten von Martin Opitz, Andreas Gryphius, Hofmann von Hofmannswaldau, Casper von Lohenstein und Angelus Silesius zu zeigen“"39. Mit Stolz betont Szyrocki am Ende des Briefes die Größe des Institutes: „Ich leite das größte germanistische Institut in Polen, wir

35 Brief von Marian Szyrocki an Günter Grass vom 20. Dezember 1985.

${ }^{36}$ Małgorzata Putra: „Katz und Maus” von Günter Grass. Versuch einer Interpretation. Wrocław 1988, 41 S. 1732; Dorota Steinhagen: Die Protagonisten der „Danziger Trilogie“ von Günter Grass. Wrocław 1988, 79 S. 1860; Marianna Draczynska: Die Frauen im „Butt “von Günter Grass. Wrocław 1981, 43 S. 1759; Zofia Szauer: Das dramatische Schaffen von Günter Grass mit besonderer Berücksichtigung des Stückes ,Die Rebellen proben den Aufstand“. Wrocław 1988, 59 S. 1731; Dorota Milewska: Das Bild der Deutschen in „Örtlich betäubt “. Wrocław 1988, 51 S. 1763; Małgorzata Nadolska: „Aus dem Tagebuch einer Schnecke“von Günter Grass als eine Schneckenbilanz. Wrocław 1988, 52 S. 1762; Maria Wiktor: Danziger Hundejahre anhand des Romans „Hundejahre" von Günter Grass. Wrocław 1988, 42 S. 1766; Urszula Wysopal: Das Problem der Schuld in Günter Grass` Roman ,Hundejahre“. Wrocław 1988, 69 S. 1765; Danuta Krynska: Günter Grass - ein Polenschriftsteller. In Anlehnung an die zwei ersten Teile der Danziger Trilogie. Wrocław 1988, 41 S. 1764; Beata Koniuszewska: Der Einakter „,Noch zehn Minuten bis Buffallo “ und das Theaterstück „Die Plebejer proben den Aufstand“ - zwei Phasen des dramatischen Schaffens von Günter Grass. Wrocław 1988, 1760.

${ }^{37}$ Marian Szyrocki: Begleitbrief, 4. März 1986.

38 Brief von Marian Szyrocki an Günter Grass vom 4. Mai 1986.

${ }^{39}$ Ebd. 
haben über 500 Studenten mit 60 Angestellten. “40 Die Antwort von Grass kam am 7. April 1986. Er könne die Einladung nicht annehmen, weil er sich nach „Abschluß der Rättin“ für ein Distanznehmen entschieden habe und ein Jahr außerhalb Europa, in Asien, verbringen möchte. ${ }^{41}$ Szyrocki bedankt sich am 29. April 1986 und informiert Grass darüber, dass der Artemis-Verlag (Zürich) ihn um eine Einführung in sein Werk gebeten hätte, was allerdings nicht zustande kam, obwohl Szyrocki das Angebot angenommen hätte und versuchen möchte, sein „Bestes zu leisten“" ${ }^{42}$ Inzwischen hatte Günter Grass die Einladung nach Breslau doch noch angenommen und zwar zu einer wissenschaftlichen Tagung, die Norbert Honsza und Marian Szyrocki veranstalteten.

Hier vielleicht einige Worte zu den anderen Aufenthalten von Günter Grass in Polen, die der Schriftsteller seit 1959 absolvierte. Zum ersten Mal kam Grass am 28. Juli 1959 nach Warschau auf Einladung der Zeitschrift „Nowa Kultura“. ${ }^{43}$ Man kann sicher gehen, dass sich hinter dieser wichtigen Einladung Andrzej Wirth, einer der Redakteure dieser Zeitschrift verbarg. Den nächsten Aufenthalt begann er am 20. April 1962. Er besuchte damals sowohl Andrzej Wirth, als auch seine Tante Anna Krause, die in Bisewo Kartuzy lebte. ${ }^{44}$ Noch in demselben Jahr stellte er einen Antrag auf ein polnisches Visum, woraufhin die SB ${ }^{45}$ Andrzej Wirth durchleuchtete. ${ }^{46}$ Am 3. Juli 1972 hat die SB ihren positiven Bescheid abgegeben. Die nächste Reise erfolgte im Jahr 1965, als das Ministerium für Kultur, Büro für Internationale Zusammenarbeit sich an das Innenministerium mit der Bitte wandte, Günter Grass nach Polen offiziell einzuladen. Grass werde von seinem Honorar für die polnische Übersetzung von Katz und Maus, die im Czytelnik-Verlag erschienen ist, die in Polen entstehenden Unkosten tragen. ${ }^{47}$ Das Büro für die Registrierung der Ausländer beim Innenministerium schrieb daraufhin an die Konsularische Abteilung des Außenministeriums, dass es keine Bedenken gegen die Einreise von Grass hätte. ${ }^{48}$ Der Schriftsteller war auch noch 1970 in Polen. Die Reise im Jahr 1972 war mit der Absicht der RIAS verbunden, einen Dokumentarfilm über Günter Grass zu drehen, woraufhin dieser Anträge für sich, für seine Ehefrau Anna Grass sowie für die beiden Kinder, Franz und Raoul Grass, stellte. In der Claris (Personalanfrage) richtete man sich bereits an einen größeren Kreis

${ }^{40}$ Ebd.

${ }^{41}$ Brief von Günter Grass an Marian Szyrocki vom 7. April 1986.

42 Brief von Marian Szyrocki an Günter Grass vom 29. April 1986.

43 Vgl. IPN BU 1268/5178. Deklaracja rejestracyjna Nr. 29783 vom 31. Juli 1959, K. 98.

44 Vgl. IPN BU 1268/5178. Deklaracja rejestracyjna N - 138203 vom 21. April 1962, K. 94-95.

${ }^{45}$ Służba Bezpieczeństwa - der Kommunistische Staatssicherheitsdiens.

46 Vgl. IPN BU 1268/5178. Interview vom 1. Juni 1962, K. 91.

47 Vgl. IPN BU 1268/5178. Pismo Ministerstwa Kultury i Sztuki. Biuro Współpracy z Zagranicą do Ministerstwa Spraw Wewnętrznych, Biura Rejestracji Cudzoziemców vom 22. Februar 1965, K. 88.

48 Vgl. IPN BU 1268/5178. Pismo do Departamentu Konsularnego MSZ vom 7. April 1965, K. 87. 
von Parteioffiziellen, die ihre Einwilligung erteilen sollten ${ }^{49}$ und sie auch erteilten. Grass war vom 27. März bis zum 8. April 1972 in Polen. Am 9. Oktober 1972 war er zum wiederholten Mal in Pommern und hielt in Gdynia einen Vortrag über sein eigenes Werk. Der Abend wurde im Rahmen der Kieler Tage (Poznaj Kilonię) in Gdynia veranstaltet. ${ }^{50}$ Vom 19. bis zum 26. Juni 1975 drehte Grass mit einem Fernsehteam einen Film über den Wiederaufbau Danzigs. Der Besuch wurde selbstverständlich „operativ gesichert“, der SB gelang es übrigens ein Skript des Films zu bekommen. Die Produktion wurde am 6. November 1975 ausgestrahlt. ${ }^{51}$

Grass besuchte Polen auch nach der Verhängung des Kriegszustandes. Noch im Mai 1983 stellte er einen Antrag auf ein Visum für die Reise nach Gdańsk-Brzezno zu seinem Verwandten Jan Krause in der Zeit vom 6. bis zum 10. Juni 1983. ${ }^{52}$ Am 8. Mai 1985 wandte sich Zrzeszenie Studentów Polskich ${ }^{53}$ an das Passbüro des Innenministeriums mit der Bitte, Günter Grass und seiner Ehefrau Uta ein Visum für die Zeit vom 17. bis zum 25. Mai 1985 zu erteilen. Der Studentenverband organisierte im Warschauer Klub „Hybrydy“ die später legendär gewordene Tagung zu Günter Grass. ${ }^{54}$ Am 19. Mai 1985 bekam das Außenministerium ein Chiffrogramm der Berliner Mission mit einer äußerst negativen Charakteristik von Grass und der Reaktion von Ute Grass auf die offensichtlich bereits erfolgte Absage des Visums:

Die Einladung von Günter Grass nach Polen erachten wir zur Zeit für nicht angebracht. G. „zeichnete sich“ durch eine besondere Bissigkeit bei den Angriffen und bei der Verleumdung der Führer des polnischen Staates und der Partei aus. Demonstrativ beteiligte er sich an keinem Treffen und an keiner Veranstaltung, die in der Mission stattgefunden haben. Aus dem Programm der Kunstakademie von Westberlin eliminierte er jegliche polnische Problematik. Er wirkt aktiv zugunsten der Umgestaltung der Akademie in ein Zentrum, das sich zum Ziel setzt, geistige Einheit der Deutschen aufrecht zu erhalten, insbesondere im Bereich der Kultur. Zur Zeit haben wir Schwierigkeiten, mit G. persönliche Kontakte anzuknüpfen, bei den Gesprächen vermittel seine Frau. ${ }^{55}$

Grass fühlte sich mit Recht brüskiert, was man aus dem Papier ablesen kann, zumal ihm und seiner Frau durch die deutsche Botschaft das Visum „versprochen“ wurde. Das Telegramm war an die Mitglieder der obersten politischen Behörde (also an das Politbüro des ZKs) geschickt worden, die Berliner Beamten nahmen

49 Vgl. IPN BU 1268/5178. Claris vom 17. Februar 1972 sowie vom 26. Februar 1972, K. 81.

50 Vgl. IPN BU 1268/5178. Naczelnik Wydziału V Biura Paszportów I do MSW vom 18. Oktober 1972, K. 67.

${ }^{51}$ Vgl. IPN BU 01228/3049. Pismo KW MO w Gdańsku do Naczelnika Wydziału VII Departamentu II Ministerstwa Spraw Wewnętrznych vom 18. September 1975, K. 3.

52 IPN BU 1268/5178. Telegraphische Anfrage der polnischen Militärmission in Berlin an das Innenministerium vom 10. Mai 1983.

53 Verband Polnischer Studenten.

54 IPN BU 1268/5178. Rada Naczelna Zrzeszenia Studentów Polskich. Komisja Zagraniczna. Pismo do Ministerstwa Spraw Wewnętrznych. Biuro Paszportów vom 8. Mai 1985, K. 42.

55 IPN BU 1268/5178. Chiffrogram Nr. 2996/II aus der Mission in Berlin vom 19. Mai 1985, K. 0035. 
die Suggestion des Ministeriums auf, Grass als offiziellen Gast des Polnischen Schriftstellerverbandes einzuladen. Aus den eventuellen Gesprächen ist nichts geworden und die nächste Gelegenheit einer Polenreise von Grass war gerade die Einladung von Marian Szyrocki und Norbert Honsza, an einer ihm gewidmeten Tagung in Karpacz teilzunehmen. Der Leiter des Departements für Wissenschaftliche Zusammenarbeit des Außenministeriums St. Klimkiewicz äußerte an die Konsularabteilung und an das Passbüro im Innenministerium keine Bedenken. ${ }^{56}$ Die Einladung der beiden erfolgte bereits am 28. Januar 1987. Noch Anfang Mai bekamen die Grass kein Visum, der Grund war folgender:

Man kann vermuten, dass G.G. beabsichtigt das erhaltene Visum zur Teilnahme an einem Seminar der Bewegung „Freiheit und Frieden“ (Wolność i Pokój) auszunutzen - er befindet sich in der Aufstellung der Teilnehmer". Da das Seminar am 10 Mai stattfinden sollte, schlug der Beamte vor die Geltung der Visa für die Zeit nach dem 15 V 1987 festzulegen. ${ }^{57}$

Der Dichter kam mit dem Auto und mit seiner Frau Ute am Montag, dem 18. Mai. Die offizielle Eröffnung der Konferenz fand im überfüllten Saal des Hauses „Krokusy“ in Karpacz statt. Ansprachen hielten Marian Szyrocki und Norbert Honsza sowie die Botschaftsrätin der Deutschen Botschaft in Warschau, Dr. Laurida Hölscher. Anwesend waren fast alle Mitarbeiter von Honsza und Szyrocki aus den beiden Wrocławer Lehrstühlen. Die ausländischen Gäste kamen aus der DDR: der Leipziger Professor Klaus Pezold (Zwibelkellerepisode), und dann aus der BRD, meistens waren das Bekannte und enge Mitarbeiter von Szyrocki und Honsza wie zum Beispiel der prominente Germanist Manfred Durzak. Die Rättin des Dichters hatte außerordentlich schlechte Rezensionen gehabt. Es erschien noch vor der Auslieferung des Romans von Grass eine Parodie „Der Grass von Günter Ratte“"58, über die Durzak sein Referat (Der parodierte Grass. Am Beispiel der Rättin) nach der Lesung des Autors am Mittwoch um 20.00 Uhr (in Abwesenheit des Dichters) hielt. Heute weiß man, dass der Dichter sehr vehement, obwohl nicht öffentlich auf das Erscheinen der Parodie reagiert hatte. ${ }^{59}$ Über diese kontroverse bundesdeutsche Rezeption der Rättin informierte der mit Norbert Honsza befreundete Germanist Graf von Nayhaus (Die Rättin im Spiegel der Rezensionen), über die Poetik des Textes die Düsseldorfer Germanistin Gertrude Cepl-Kaufmann (Über die Rättin). Andere Prominente Gäste aus der Bundesrepublik waren der Lyrikforscher Hanspeter Brode (Das Polenbild in den Romanen von Günter

56 IPN BU 1268/5178. Schreiben vom 20. März 1987, K. 28.

57 IPN BU 1268/5178. Chiffrogramm Nr. 1740/II aus der Mission in Berlin vom 1. Mai 1987, K. 12.

58 Günter Ratte, Der Grass. Das literarische Bubenstück. Eine geheimnisvolle Parodie, die unter die Gürtellinie greift - und ihre Hintergründe. Edition BOD. Herausgegeben von Vito von Eichborn. 134 S., ISBN: 3-8334-6550-6, Norderstedt, November 2006.

59 „Der Herausgeber (Vito von Eichborn - W.K.) berichtet in seinem Vorwort, daß man im Luchterhand Verlag den aufgeregten Günter Grass nur mit Mühe von dem angesichts der Rechtslage völlig aussichtlosen Versuch abbringen konnte, gegen diese Parodie gerichtlich vorzugehen." Vgl. http://www.geowis.de/product_info.php?products_id=239. [Zugriff am 26. Januar 2014]. 
Grass von der Blechtrommel bis zur Rättin), der Biograph von Grass und Herausgeber seiner Werke in 10 Bänden Volker Neuhaus (Eine Information über die Günter-Grass-Ausgabe), der mit Marian Szyrocki kooperierende Barockforscher Jörg-Ulrich Fechner (,,Vorfindlichkeit". Zur Poetik einer sekundären Imagination in Günter Grass MARIAZUEHREN und MUELL UNSER $)^{60}$ sowie der bekannte Schöpfer der interkulturellen Germanistik Alois Wierlacher (Großmutter Anna und die schwarze Köchin. Zur Thematik des Essens in Grass' Die Blechtrommel). Im Protokollband, der 1990 erschien, wurden Beiträge von Durzak, Brode und Fechner nicht veröffentlicht. Neben den genannten erschienen aber Aufsätze polnischer Referenten der Tagung: Norbert Honsza (Sisyphos verhöhnt die Götter (Die Rättin)), Marian Szyrocki (Adebar. Offene Gedichtinterpretation), Zbigniew Światłowski (Apperzeptionsverweigerung als Thema der modernen Literatur), Jan Miziński (Eine katastrophale Vision. Zu einigen Aspekten des Romans Die Rättin), Henryka Sztumowska (Der politisierende Schriftsteller Günter Grass), Leszek Żyliński (Günter Grass' nationale Sendung (Publizistik)). Im Mittelpunkt stand also der damals erschienene Roman Die Rättin sowie die Fragen, die weniger mit der Poetik, als mit der Soziologie und Wirkung des Werkes von Grass verbunden waren. Nach der Tagung hat Günter Grass unter der fachkundigen Leitung Breslau besichtigt und u.a. Norbert Honsza in dessen Haus besucht. ${ }^{61}$ Grass war zufrieden, zumal er sich in der Mitte seiner (nur gedämpft kritischen) Bewunderer fand. ${ }^{62}$ Nach der Tagung begannen Szyrocki und Grass sich zu duzen, beabsichtigte der Germanist noch ein Kolloquium zu veranstalten, diesmal in der Reihe seiner berühmt gewordenen Karpaczer Tagungen zur Barockliteratur: „Was würdest Du zu einer Barockkonferenz über das Thema „Das Treffen in Telgte“ sagen? Die Ba-

${ }^{60}$ Brief von Jörg-Ulrich Fechner an den Verf. vom 16. März 2014: „Mein Grass-Beitrag in Karpacz blieb ungedruckt. Herr Neuhaus wollte ihn nicht gern, weil Grass ihm wohl gesagt hatte, dass ich eins seiner kleineren Werke zu Unrecht in den Mittelpunkt gestellt hätte. - Der Vortrag stammte noch von meiner Schreibmaschine. Wo er in meinen Unterlagen sich befindet, weiß ich im Augenblick nicht und müsste an vielen Stellen danach suchen."

Leonard Forster und Marian Szyrocki waren miteinander sehr vertraut und auch persönlich eng verbunden. Wir trafen uns ja regelmäßig in Wolfenbüttel zu den Sitzungen der Barock-Kommission. Während eines gemeinsamen Gastsemesters in Kiel machten wir drei mit den Kieler Kollegen ein Oberseminar über das Treffen in Teltge und beleuchteten dabei besonders die Bezüge, die Grass dabei auf Anregungen von Marian und von Herrn Schöne zur deutschen Barockliteratur verarbeitet und literarisch gestaltet hatte. Aus diesen Seminarsitzungen ist keine gedruckte Arbeit geworden.“ Es konnte sich um das Jahr 1981 handeln. Lenard Foster war damals Gastprofessor in Kiel. Grass besuchte Kiel am 21. November 1981 und las aus seinen Werken. Im „Semester. Zeitung an der Christian-Albrechts-Univerersität zu Kiel“ Nr. 14. Januar 1981, S. 4-5 wurden zwei Interviews von Grass: mit Lenard Foster und Wofgang Kehl abgedruckt.

${ }^{61}$ Der Mensch wird an seiner Dummheit sterben. Günter-Grass-Konferenz. Karpacz 17.-23. Mai 1987. Hrsg. von Norbert Honsza, Jerzy Łukosz, Marian Szyrocki. Wrocław 1990.

${ }^{62}$ Im Brief vom 19. Juni 1987 schrieb er an Marian Szyrocki und Norbert Honsza: „Auch daß es so heiter zugehen konnte zwischen Autor und Germanisten, zählt für mich als schöne Ausnahme, das soll bedankt sein." 
rockforscher würden zum Teil über ihre Forschungsvorhaben berichten. Im Mittelpunkt der Konferenz sollte aber das „Treffen“ und das Barockkapitel im „Butt“ stehen, verbunden mit Deiner Lesung. "63 Günter Grass hat aber bereits für 1988 eine Reise in die Sowjetunion sowie die Teilnahme an der Deutschen Kulturwoche in Polen ${ }^{64}$ zugesagt und bittet Szyrocki, die geplante Konferenz für den Anfang des Jahres 1989 zu verlegen. Grass erinnerte sich gern an die Karpaczer Tagung: „Die Tage unter der zumeist neblig verhangenen Schneekoppe sind Ute und mir noch immer in guter Erinnerung." "65 Noch im August 1988 bekräftigt er seine Absage an der geplanten Tagung, ihn interessiert aber das, was er in Krumhübel 1987 sehr wohl beobachten konnte: die erschreckenden Ausmaße des Waldsterbens:

Doch habe ich mir fest vorgenommen, im Verlauf des nächsten Jahres im Riesengebirge sterbenden Wald und im Detail tote Bäume zu zeichnen. Bei dieser Gelegenheit ließe sich dann, wenn Du magst, eine Lesung in Breslau arrangieren und natürlich ein Treffen in Karpacz (Vielleicht könntest Du mich als Rübezahl an die richtigen Stellen führen und auch noch einen Förster auftreiben, der mir Genaues über den Zustand des Waldes sagt). ${ }^{66}$

Gedrängt durch diverse Termine bittet Grass endlich für eine Einladung im Frühjahr 1990 und wenn das nicht ginge, dann „die Woche vom Montag, den 6.11. (Anreisetag nach Wroclaw und Lesung und Diskussion am Abend) bis Samstag, dem 11 November (Abreisetag)." Seine Hauptabsicht bleiben aber nach wie vor Zeichnungen zum Waldsterben: „Im Spätherbst jedoch, etwa während der ersten Novemberhälfte, befürchte ich schlechtes Wetter, so daß das notwendige Freilichtzeichnen buchstäblich ins Wasser fallen könnte." ${ }^{\text {"67 }}$ Am Rande sei bemerkt, dass die Zeichnungen von Günter Grass zum Thema Waldsterben Totes Holz. Ein Nachruf 1990 in Göttingen erschienen sind.

Als Epilog dieser Freundschaft zwischen Szyrocki und Grass kann man den Doktor h.c. für Günter Grass in Posen erwähnen, der durch den bekannten Germanisten Hubert Orłowski angeregt wurde. Marian Szyrocki schrieb das Gutachten für dieses Verfahren: Die wichtigste Frage, die Grass in seinem vielleicht schönsten Werk Das Treffen in Telgte gestellt hatte, bezieht sich auf die Rolle des Schriftstellers in einer Welt, die durch Kriege, Bürgerkriege und Totalismen geprägt wird. Die in das 17. Jahrhundert verlegte Diskussion über das Verhältnis der Autoren zu

63 Brief von Marian Szyrocki an Günter Grass vom 17. Juni 1987.

${ }^{64}$ Die sehr aufwendige Deutsche Kulturwoche wurde vom Goethe-Institut im Oktober 1988 veranstaltet. Damals fanden 39 Veranstaltungen statt, es beteiligten sich 250 Künstler und Wissenschaftler aus der Bundesrepublik Deutschland. Besonders wichtig war die Podiumsdiskussion von Günter Grass mit Adam Michnik und Jan Józef Lipski im Studentenklub „Hybrydy“. Siehe: Jürgen Vietig: Die Erinnerungen fahren mit. Deutsche Kulturwoche in Polen: Hochgespannte Erwartungen und auch Mißverständnisse, in: „Süddeutsche Zeitung“ vom 24. Oktober 1988. Siehe auch: Stefan Dietrich: Der Ofen glüht schon, in: FAZ vom 2. November 1988.

65 Brief von Günter Grass an Marian Szyrocki vom 19. November 1987.

${ }^{66}$ Brief von Günter Grass an Marian Szyrocki vom 26. August 1988.

${ }^{67}$ Brief von Günter Grass an Marian Szyrocki vom 14. März 1989. 
den geschichtlichen Ereignissen zeitigt die wichtigste Diagnose, die sich auf die friedensstiftende Rolle des Schriftstellers beläuft. ${ }^{68}$

\title{
Literatur
}

\section{Primärliteratur}

Briefwechsel Günter Grass-Marian Szyrocki. Sign. 9047. Akademie der Künste. Berlin.

Briefwechsel Günter Grass-Marian Szyrocki. In: Archiv Marian Szyrocki. Bibliothek des Instituts für Germanische Philologie der Universität Breslau. Wrocław.

Dokumente des Instituts für Nationales Gedenken (Instytut Pamięci Narodowej). Sign. IPN BU $1268 / 5178$.

Grass, Günter (1977): Der Butt. Roman. Darmstadt und Neuwied.

\section{Sekundärliteratur}

Szyrocki, Marian (1959): Der junge Gryphius. Berlin.

Szyrocki, Marian (1974): Martin Opitz. Zweite, überarbeitete Auflage. München.

\begin{abstract}
s
Die Kontakte von Günter Grass zu Germanisten aus Polen, insbesondere zu Marian Szrocki, dem ehemaligen Direktor des Instituts für Germanistik der Universität Wrocław, bilden einen wichtigen, wissenschaftsgeschichtlichen Bestandteil der Polen-Germanistik nach 1945. Der Aufsatz beleuchtet, sich auf bislang unveröffentlichte Quellen (Briefe, Akten des IPN - des Instituts für Nationales Gedenken) stützend, die Wechselbeziehungen zwischen dem Blechtrommel-Autor und dem Barockforscher Szyrocki. Nicht ausgelassen werden komplizierte und prekäre Aspekte dieser Bekanntschaft. Durch diese Darstellung werden Mechanismen einer Fachrichtung (in diesem Fall der Germanistik) beleuchtet, die in einem Regimestatt funktionieren muss. Parallel dazu erfährt man auch von den zwischenmenschlichen Wechselbeziehungen der im Titel angeführten Protagonisten.
\end{abstract}

Schlüsselwörter: Günter Grass, Marian Szyrocki, Wissenschaftsgeschichte, Germanistik, Barock, IPN

${ }^{68}$ Marian Szyrocki: Gutachten über Günter Grass vom 24. Juni 1989. In: Archiv Marian Szyrocki. Bibliothek des Instituts für Germanische Philologie der Universität Breslau: „Najważniejsze jednak pytanie, jakie Grass postawił w najpiękniejszym być może ze swoich dzieł, „Spotkanie w Telgte" odnosi się do roli pisarza w świecie zdominowanym przez wojny, wojny domowe i totalizmy. Przeniesiona w wiek XVII problematyka pisarza wobec wydarzeń historycznych sprowadza się nie tylko do formuły realizmu, ale i do diagnozy: pisarz swoim dziełem ma stanowić pokój.” 


\title{
Marian Szyrocki and Günter Grass
}

Günter Grass's relations with Polish German specialists, not least with Marian Szyrocki, former head of the Department of German Studies of the University of Wrocław, constitute a relevant, historic and scientific element of Polish German studies after the year 1945. The article, based on unpublished sources (letters, files of the National Institute of Remembrance), depicts the relations between the author of The Tin Drum and Szyrocki. The author of this article does not omit the complicated and critical aspects of the relations between the German writer and the Polish German specialist. Furthermore, the objective of this paper is to present the mechanisms of a scientific field (in our case German studies) functioning in a totalitarian state. The author also takes into account the interpersonal relations between Grass and Szyrocki.

Keywords: Günter Grass, Marian Szrocki, history of science, German studies, baroque, IPN

\author{
Wojciech Kunicki \\ Uniwersytet Wrocławski \\ Instytut Filologii Germańskiej \\ Pl. Nankiera $15 \mathrm{~b}$ \\ 50-140 Wrocław \\ Polen \\ E-Mail: wojciech.kunicki@uwr.edu.pl
}

